\title{
Correction to: On Families of Periodic Orbits in the Restricted Three-Body Problem
}

\section{Seongchan $\mathrm{Kim}^{1}$}

Published online: 28 October 2019

() Springer Nature Switzerland AG 2019

\section{Correction to:}

\section{Qualitative Theory of Dynamical Systems (2019) 18:201-232 https://doi.org/10.1007/s12346-018-0288-X}

The following replaces page 203, line 27 to the end of the introduction of [9].

In this paper, we take a closer look at Euler's problem of two fixed centers, which was first introduced by Euler [4,5]. He considered this problem as a starting point to study the PCR3BP. The describing Hamiltonian is obtained from the PCR3BP by forgetting the rotating term (Fig. 1):

$$
H_{\text {Euler }}(q, p)=\frac{1}{2}|p|^{2}-\frac{1-\mu}{|q-E|}-\frac{\mu}{|q-M|} .
$$

This system describes the dynamics of the satellite attracted by two masses of mass $1-\mu$ and $\mu$ that are fixed at $E$ and $M$. If $\mu=1 / 2$ and $\mu$ is viewed as a charge instead of a mass, then this system can also be seen as describing the motion of an electron attracted by two protons, as in the hydrogen molecule, see [10].

Applying Poincaré's strategy to Euler's idea naturally leads to the question: Can one find periodic orbits in the PCR $3 B P$ by homotoping periodic orbits in the Euler problem? If it is possible, how different are they from the ones obtained from periodic orbits in the rotating Kepler problem? In this paper, we study this question by computing the invariants $\mathcal{J}_{1}$ and $\mathcal{J}_{2}$, which recently introduced by Cieliebak-Frauenfelder-van Koert in [2], for periodic orbits in the Euler problem. For definition and properties of the invariants, see Sect. 2. These invariants serve as obstructions to the existence of a homotopy of periodic orbits. We then compare them with the ones for the rotating Kepler problem provided in [6].

The original article can be found online at https://doi.org/10.1007/s12346-018-0288-x.

Seongchan Kim

seongchan.kim@math.uni-augsburg.de

1 Universität Augsburg, Universitätsstrasse 14, 86159 Augsburg, Germany

Birkhäuser 
The PCR3BP

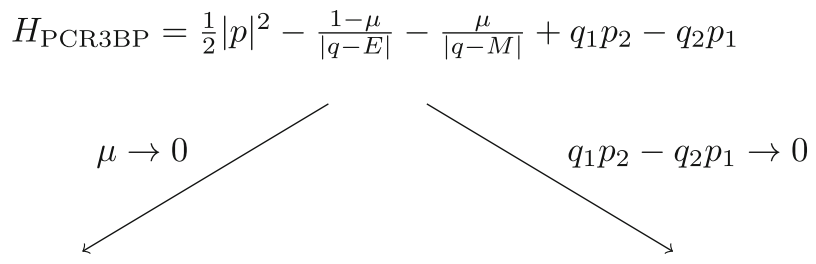

The rotating Kepler problem

The Euler problem

$H_{\mathrm{RKP}}=\frac{1}{2}|p|^{2}-\frac{1}{|q|}+q_{1} p_{2}-q_{2} p_{1} \quad H_{\text {Euler }}=\frac{1}{2}|p|^{2}-\frac{1-\mu}{|q-E|}-\frac{\mu}{|q-M|}$

Fig. 1 Two special cases of the PCR3BP

Theorem Let $\gamma^{R K P}$ and $\alpha^{\text {Euler }}$ be periodic orbits in the rotating Kepler problem and Euler problem, respectively, which are freely homotopic. Then, the CieliebakFrauenfelder-van Koert invariants agree on $\gamma^{R K P}$ and $\alpha^{\text {Euler }}$.

Remark Recall that the Conley-Zehnder indices of periodic orbits in the rotating Kepler problem and Euler problem agree [1,7]. In view of this and the previous theorem, we expect that there might be more intrinsic relationship between the two problems. In other words, there might be a hope that during a homotopy of periodic orbits from the Euler problem via the PCR3BP to the rotating Kepler problem, no birth-death bifurcation occurs, see Fig. 2.

Remark The $\mathcal{J}_{1}, \mathcal{J}_{2}$ invariants are defined for periodic orbits near either $E$ or $M$. In [3], Cieliebak-Frauenfelder-Zhao define new invariants for periodic orbits which encircle both primaries. An explicit example for such invariant is provided in [8].

The Euler problem

The rotating Kepler problem

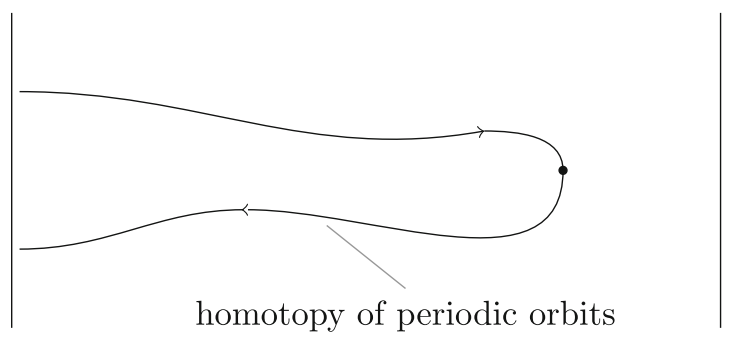

Fig. 2 A possible birth-death bifurcation. Due to the result of the theorem, we expect that this does not happen 


\section{Correction to Proposition 2.13}

There is a gap in the last paragraph in page 212 and in the proof of Proposition 2.13. The assertions hold true for brake-brake orbits and brake-collision orbit, but not for collision-collision orbits.

If $K$ is either a brake-brake orbit or a brake-collision orbit, then any two perturbed curves only differ from each other by orientation and the number of exterior loops and crossings through a triple point, see Fig. 7 in [9]. By definition, these two perturbations have the same $J^{+}$. Thus, we can define the $J^{+}$-invariant $K$ by the one of $\widetilde{K}$, i.e.,

$$
J^{+}(K):=J^{+}(\widetilde{K})
$$

This together with the fact that $w_{0}(K)^{2}$ does not change by small perturbations as well implies that the definitions $\mathcal{J}_{i}(K):=\mathcal{J}_{i}(\widetilde{K}), i=1,2$, are also well defined.

Let $K$ be a collision-collision orbit. Note that we have $w_{0}(K)^{2}=0$ or 4, depending on perturbations. Likewise, the $J^{+}$invariant might depend on perturbations as well and hence in this case (1.1) is not well defined. However, the $\mathcal{J}_{1}$ invariant is independent of perturbations. For example, look at Fig. 3 which illustrates two possible perturbations of $K$. For the left perturbation, we have $w_{0}(K)^{2}=4$. During a Stark-Zeeman homotopy from $K$ to a circle, we encounter only an inverse self-tangency which implies that the perturbation has $J^{+}=0$. For the right one, we have $w_{0}(K)^{2}=0$. In this case, we have a direct self-tangency during a Stark-Zeeman homotopy, and hence, the perturbation has $J^{+}=2$. In any case, we have $\mathcal{J}_{1}=2$. The assertion for $\mathcal{J}_{2}$ can be shown in a similar manner. Thus, we also define $\mathcal{J}_{i}$ of $K$ as $\mathcal{J}_{i}(K):=\mathcal{J}_{i}(\widetilde{K})$, $i=1,2$.

Proposition 2.13 The two quantities $\mathcal{J}_{1}$ and $\mathcal{J}_{2}$ are invariants for Stark homotopies.

Proof Let $\left(K^{s}\right)_{s \in[0,1]}$ be a Stark homotopy. Suppose that $K^{s_{0}}$ is one of the distinguished orbits for some $s_{0} \in(0,1)$. Since $K^{s_{0} \pm \varepsilon}$ are generic immersions and small perturbations of $K^{s_{0}}$, provided that $\varepsilon>0$ is small enough, we have $\mathcal{J}_{i}\left(K^{s_{0}-\varepsilon}\right)=\mathcal{J}_{i}\left(K^{s_{0}+\varepsilon}\right)$, $i=1,2$. This completes the proof of the proposition.
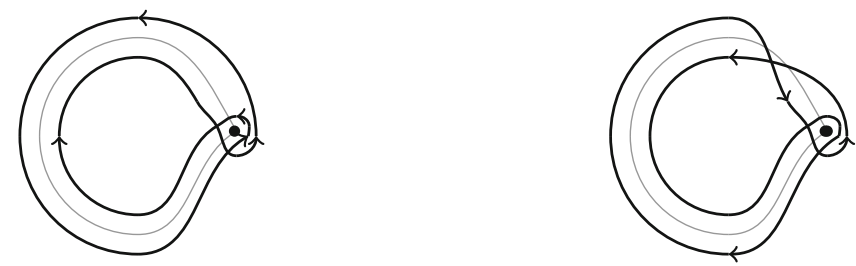

Fig. 3 Two perturbations of a collision-collision orbit. We have $w_{0}(K)^{2}=4$ for the one in the left-hand side and $w_{0}(K)^{2}=0$ for the right one 


\section{Correction to Proposition 2.14}

The assertion on page 214 , line 15 , "Since $L$ is a $2-1$ covering, it follows that the number of quadruple points along $\gamma_{1}$ equals that of $\gamma$ " is certainly incorrect: Let $\gamma$ be a brake-brake orbit with $N$ quadruple point. Since it has even winding number, the preimage $L^{-1}(\gamma)$ has two connected components $\gamma_{1}, \gamma_{2}$. Since $L$ is 2-to- $1, L^{-1}(\gamma)$ has $2 N$ quadruple points, but some of them might be intersections of $\gamma_{1}, \gamma_{2}$. It is not easy to determine the number of such intersections for an arbitrary $\gamma$, so Proposition 2.14 and the paragraph preceding it should be replaced by the following.

We conclude this section with the following proposition which provides formulas for the $\mathcal{J}_{1}$ invariants of brake-brake orbits and brake-collision orbits and $\mathcal{J}_{2}$ invariants for brake-collision orbits which will be used to prove the main result in Sect. 4 in [9]. The $\mathcal{J}_{2}$ invariant of brake-brake orbits in the Euler problem will be given later.

Proposition 2.14 Let $\gamma$ be a brake-brake orbit or brake-collision orbit. Assume that all intersection points of $\gamma$ are quadruple and the number of quadruple points equals $N$. Then, we have

$$
\mathcal{J}_{1}(\gamma)= \begin{cases}2 N & \text { if } \gamma \text { is a brake-brake orbit } \\ 2 N+1 / 2 & \text { if } \gamma \text { is a brake-collision orbit }\end{cases}
$$

and

$$
\mathcal{J}_{2}(\gamma)=4 N \text { if } \gamma \text { is a brake-collision orbit. }
$$

Proof Let $\gamma$ be as in the assertion. By the previous argument, one can generically homotope a brake-brake orbit to a circle which does not encircle the origin and a brake-collision orbit can be generically homotoped to a circle which encircles the origin. Therefore, the winding number of a brake-brake orbit around origin is given by zero and that of a brake-collision orbit equals either plus or minus one. Note that after a small perturbation, each quadruple point gives rise to four double points of a generic immersion. During a homotopy, it leads to one crossing through a direct selftangency and one crossing through an inverse self-tangency (possibly with a finite number of crossings through a triple point). Since the perturbation of $\gamma$ is generically homotoped to a circle, we conclude that $J^{+}(\gamma)=2 N$. This proves the assertion for the $\mathcal{J}_{1}$ invariants.

If $\gamma$ is a brake-collision orbit, then the pre-image consists of a single orbit consisting of inverse self-tangencies. By definition of the map $L$, the number of quadruple points along $L^{-1}(\gamma)$ is given by $2 N$. This proves the formulas for the $\mathcal{J}_{2}$ invariants and completes the proof of the proposition.

\section{Correction to Theorems 4.8 and 4.10}

The sentence in page 229, line 7 should be deleted. We restate and prove Theorem 4.8 . 
Theorem 4.8 For the $T_{k, l}$-torus family in the $S$-or $S^{\prime}$-region in the Euler problem, we have

$$
\mathcal{J}_{1}\left(T_{k, l}\right)= \begin{cases}2 k l-k-l+1 & \text { if } k+l \text { is odd } \\ (k l-k-l+2) / 2 & \text { if } k+l \text { is even }\end{cases}
$$

and

$$
\mathcal{J}_{2}\left(T_{k, l}\right)=k l-k-l+1
$$

Proof In view of Propositions 2.14 and 4.4, it remains to prove the formula of $\mathcal{J}_{2}$ invariant for the case that $k+l$ is odd. We only consider the case that $k$ is even and $l$ is odd. The other case can be proved in a similar way.

Let $\gamma$ be a brake-brake orbit of period T. As in the proof of Proposition 4.4, we only consider the half $\left.\gamma\right|_{[0, T / 2]}$, which will be denoted again by $\gamma$, and then, every self-intersection point is a double point. Without loss of generality, we may assume that $T=2$. We also parametrize $K$ so that the braking point with $q_{2}>0$ is the initial point and hence the braking point with $q_{2}<0$ is the end point.

We assign each double point of $\gamma$ a rotation number as follows. Let $p$ be a double point, and we then find $0<t_{0}<t_{1}<1$ such that $\gamma\left(t_{0}\right)=\gamma\left(t_{1}\right)=p$. The rotation number $\operatorname{rot}(p)$ is defined as the winding number of the tangent vector $\gamma^{\prime}(t)$ for $t \in$ $\left[t_{0}, t_{1}\right]$. It is obvious that this number is an integer.

Recall that since the winding number of $\gamma$ is even, the pre-image $\tilde{\gamma}:=L^{-1}(\gamma)$ consists of two connected components $\gamma_{1}, \gamma_{2}$. We fix a double point $p$ of $\gamma$ and $0<t_{0}<t_{1}<1$ as above. The pre-image $L^{-1}(p)$ is a pair of double points $p_{1}, p_{2}$ of $\tilde{\gamma}$. As already pointed out, there are two possibilities: one is that $p_{1}, p_{2}$ are intersections between $\gamma_{1}, \gamma_{2}$, and the other is that $p_{1}, p_{2}$ is a double point of $\gamma_{1}, \gamma_{2}$, respectively. In order to determine the $\mathcal{J}_{2}$ invariant of $\gamma$, we have to count double points of $\gamma_{1}$ (or equivalently of $\gamma_{2}$ ), and hence, we need the second scenario. Note that since $L(z)=z^{2}$, it is the case if and only if $\operatorname{rot}(p)$ is even.

We claim that the number of double points of $\gamma_{1}$ is given by

$$
\frac{1}{2}(k-1)(l-1)
$$

from which the assertion of the theorem follows. To this end, we take the approach of the proof of Proposition 4.4. Suppose that $t_{0} \in(0,1)$ (recall that we have assumed that $T=2$ ) represents a double point of $\gamma$ of even rotation number. We abbreviate $\left(\lambda_{0}, v_{0}\right)=\left(\lambda\left(t_{0}\right), v\left(t_{0}\right)\right)$ and find that

$$
t=t_{1}, \frac{2}{k}+t_{1}, \frac{4}{k}+t_{1}, \ldots, \frac{k-2}{k}+t_{1}
$$

at which $\lambda=\lambda_{0}$, where $t_{1} \in(0,1 / 2 k)$ and that

$$
t=t_{2}, \frac{1}{l} \pm t_{2}, \frac{2}{l} \pm t_{2}, \ldots, 1-t_{2}
$$


at which $v=v_{0}$, where $t_{2} \in(0,1 / 2 l)$. Proceeding as in Claim 1 of the proof of Proposition 4.4, one can show that $t_{0}$ is of the form

$$
\frac{\alpha k+2 \beta l}{2 k l}, \quad \alpha, \beta \in \mathbb{Z}
$$

Note that

$$
\#\left\{\frac{\alpha k+2 \beta l}{2 k l} \in[0,1] \mid \alpha, \beta \in \mathbb{Z}\right\}=k l+1 .
$$

As before, not every such point represents a double point. We set

$$
A=\left\{\frac{i}{k} \mid i=0,1, \ldots, k\right\}
$$

and

$$
B=\left\{\frac{j}{2 l} \mid j=0,1, \ldots, 2 l\right\} .
$$

The elements in $A$ correspond to the maximum of $|\lambda|$, and it is obvious that they do not represent double points. Among $2 l+1$ elements in $B$, the $(l+1)$ elements $j / 2 l$, $j=0,2, \ldots, 2 l$, correspond to the maximum of $|v|$, which do not represent double points. The remaining $l$ elements $j / 2 l, j=1,3, \ldots, 2 l-1$, correspond to $v=0$. Here, we have used, as in the proof of Proposition 4.4, the symbol $v$ for $v+\pi$ for sake of convenience. Recall that $v=0$ represents the negative $q_{1}$-axis on which there exist a single point and $(l-1) / 2$ double points. Since $L(z)=z^{2}$ and $\gamma$ is $q_{1}$-symmetric, $\gamma_{1}$ is $q_{2}$-symmetric. This in particular implies that a single point and the $(l-1) / 2$ double points of $\gamma$ on the negative $q_{1}$-axis represent a single point and $(l-1) / 2$ double points of $\gamma_{1}$ on the $q_{2}$-axis. Proceeding as in Claim 2 of the proof of Proposition 4.4, we find the number of double points of $\gamma$ of even rotation number equals

$$
\frac{1}{2}(k l+1-(k+1)-(l+1)+2)=\frac{1}{2}(k-1)(l-1) .
$$

This proves the claim and completes the proof of the theorem.

The following line replaces page 231, lines 1-2 of the paper: In a similar way, we find that

$$
\mathcal{J}_{2}\left(T_{(k+l) / 2,(k-l) / 2}^{\text {Euler }}\right)=1-k+\frac{k^{2}-l^{2}}{4}=\mathcal{J}_{2}\left(T_{k, l}^{\mathrm{RKP}}\right) .
$$

We are now in a position to restate Theorem 4.10.

Theorem 4.10 Let $\gamma^{R K P}$ and $\alpha^{\text {Euler }}$ be torus-type orbit in the rotating Kepler problem and in the Euler problem, respectively, of same knot type. They have the same $\mathcal{J}_{1}$ and $\mathcal{J}_{2}$ invariants. 
The last sentence in the paragraph preceding to Acknowledgement should be deleted.

\section{References}

1. Albers, P., Fish, J.W., Frauenfelder, U., van Koert, O.: The Conley-Zehnder indices of the rotating Kepler problem. Math. Proc. Camb. Philos. Soc. 154(2), 243-260 (2013)

2. Cieliebak, K., Frauenfelder, U., van Koert, O.: Periodic orbits in the restricted three-body problem and Arnold's $J^{+}$-invariant. Regul. Chaotic Dyn. 22(4), 408-434 (2017)

3. Cieliebak, K., Frauenfelder, U., Zhao, L.: $J^{+}$-invariants for planar two-center Stark-Zeeman systems (2019). arXiv:1910.04570

4. Euler, L.: Un corps étant attiré en raison déciproque quarrée des distances vers deux points fixes donnés. Mémoires de l'Acad. de Berlin, pp. 228-249 (1760)

5. Euler, L.: De motu corporis ad duo centra virium fixa attracti. Novi Commentarii Academiae Scientiarum Imperialis Petropolitanae 10, 207-242 (1766)

6. Kim, J., Kim, S.: $J^{+}$-like invariants of periodic orbits of the second kind in the restricted three-body problem. J. Topol. Anal. (online ready)

7. Kim, S.: Dynamical convexity of the Euler problem of two fixed centers. Math. Proc. Camb. Philos. Soc. 165(2), 359-384 (2018)

8. Kim, S.: Arnold's $J^{+}$-invariants and the lemniscate motions in Euler's two-center problem (in preparation) (2019)

9. Kim, S.: On families of periodic orbits in the restricted three-body problem. Qual. Theory Dyn. Syst. 18(1), 201-232 (2019)

10. Pauli, W.: Über das Modell des Wasserstoffmolekülions. Ann. Phys. 68, 177-240 (1922)

Publisher's Note Springer Nature remains neutral with regard to jurisdictional claims in published maps and institutional affiliations. 